\title{
Towards the zero-surface-tension limit in granular fingering instability
}

\author{
XIANG CHENG*, LEI XU๋, AARON PATTERSON, HEINRICH M. JAEGER AND SIDNEY R. NAGEL \\ James Franck Institute and Department of Physics, The University of Chicago, Chicago, Illinois 60637, USA \\ ${ }^{\dagger}$ Present address: Department of Physics, Harvard University, Cambridge, Massachusetts 02138, USA \\ *e-mail: xcheng@uchicago.edu
}

The finger-like branching pattern that occurs when a less viscous fluid displaces a more viscous one confined between two parallel plates has been widely studied as a classical example of a mathematically tractable hydrodynamic instability ${ }^{1-3}$. Fingering in such Hele-Shaw geometries has been generated not only with newtonian fluids ${ }^{4-6}$ but also with various non-newtonian fluids ${ }^{7-9}$ including fine granular material displaced by gas, liquid or larger grains ${ }^{10-15}$. Here, we study a granular Hele-Shaw system to explore the zero-surface-tension property of granular 'fluids ${ }^{16}$. We demonstrate that the grain-gas interface exhibits fractal structure and sharp cusps, which are associated with the hitherto-unrealizable singular hydrodynamics predicted in the zero-surface-tension limit of normal fluid fingering ${ }^{2,17-23}$. Above the yield stress, the scaling for the finger width is distinct from that for ordinary fluids, reflecting unique granular properties such as friction-induced dissipation as opposed to viscous damping ${ }^{24-27}$. Despite such differences, the dimension of the global fractal structure and the shape of the singular cusps on the interface agree with the theories based on simple laplacian growth of conventional fluid fingering in the zerosurface-tension limit ${ }^{2,17-23}$.

Our granular material consists of spherical glass beads with diameters for separate experiments of: $d=54 \pm 10 \mu \mathrm{m}, 107 \pm 19 \mu \mathrm{m}$ and $359 \pm 61 \mu \mathrm{m}$. The material is loaded into a traditional radial Hele-Shaw cell consisting of two 1.9-cm-thick circular glass plates of diameter $L$. In most experiments, $L=50.8 \mathrm{~cm}$, but smaller plates with $L=25.3 \mathrm{~cm}$ and $17.5 \mathrm{~cm}$ are also used. The gap between the plates, $b$, is uniform and in different experiments was varied between $0.25 \mathrm{~mm}$ and $1.14 \mathrm{~mm}$. The two plates are maintained at a fixed gap with spacers and clamps around the perimeter. The granular material driven by the displacing gas can flow freely out of the cell boundary. The system was vibrated so that the material between the plates was uniformly dense with a packing fraction near that of random close-packing. To drive off any humidity, which can cause cohesion between particles, the beads are baked under vacuum before use. We estimated the cohesive force between beads from the angle of the repose of the material ${ }^{28}$. The ratio between the cohesive force and the weight of beads is $6.9 \%$ for $54 \mu \mathrm{m}$ beads and $3.5 \%$ for $107 \mu \mathrm{m}$ beads. By calculating the surplus surface energy resulting from this cohesive force, we find that the effective surface tension of the granular material is about $10^{-6} \mathrm{~mJ} \mathrm{~m}^{-2}$ for both $54 \mu \mathrm{m}$ and $107 \mu \mathrm{m}$ beads, which is seven orders of magnitude smaller than that of normal fluids such as water $\left(72.8 \mathrm{~mJ} \mathrm{~m}^{-2}\right)$ or ethyl alcohol $\left(22.3 \mathrm{~mJ} \mathrm{~m}^{-2}\right)$.
Pressurized nitrogen, with pressure $\Delta P$ higher than ambient pressure, is blown through a $2 \mathrm{~mm}$ hole in the centre of the bottom plate. The patterns formed are recorded by a high-speed camera (Phantom V7.1) at 2,000 frames per second or by a high-resolution still camera. To study the dynamics of granular fingering near the yield stress of the granular material, corresponding to a threshold pressure $\Delta P_{\text {th }}$, we also conducted our experiment under small constant flow rate conditions using a syringe pushed by a speedcontrolled motor. With a flow rate of $22.9 \pm 0.1 \mathrm{ml} \mathrm{s}^{-1}$ at the smallest gap thickness used, $b=0.25 \mathrm{~mm}$, this covered $0.04 \%$ of the entire plate area per second. The patterns grow by stick-slip motion. When the gas pressure is above $\Delta P_{\text {th }}$, patterns grow leading to a drop in pressure so that finger growth halts. Then the gas pressure builds up until the fingers resume their expansion. This method forces the pattern to grow under conditions such that the pressure is always close to $\Delta P_{\text {th }}$.

Figure $1 \mathrm{a}, \mathrm{b}$ shows viscous fingering patterns in the granular Hele-Shaw experiments. The shape and dynamics of the pattern depend on the driving pressure, $\Delta P$. When below the yield stress, patterns do not form. Above the yield stress, there are two stages of growth that can be seen by measuring the size of the fastest moving finger. In the early stage, the finger grows at a slow, approximately constant, speed as seen in Fig. 1c. In this regime, shown in Fig. 1a, we can see a pattern similar to those in fluids. In the late stage, shown in Fig. 1b, possibly influenced by the boundary, the growth is accelerated and the patterns grow wildly. In this regime, we see the merging of fingers and the pinch-off of fingers from the main structure. Such phenomena are rare in fluid fingering. Figure 1c shows that the two stages are connected by a sharp kink at low $\Delta P$ that becomes smoother as the pressure increases. We focus here on the patterns in the early stage.

Figure $2 \mathrm{a}-\mathrm{c}$ shows the patterns of fingers at different $\Delta P$. The patterns are sharper and more ramified at low pressure and become smoother and more circular at high pressure. This is opposite to what happens in newtonian fluids where the width of a finger, $W$, depends on the capillary number $\mathrm{Ca}$ as

$$
W=\pi b \sqrt{1 / \mathrm{Ca}}=\pi b \sqrt{\sigma /(\eta V)},
$$

where $\sigma$ is the surface tension acting across an interface, $\eta$ is the viscosity of the more viscous fluid (ignoring the viscosity of the less viscous fluid as it is usually much smaller) and $V$ is the local interface velocity ${ }^{2,5}$. The competition between viscous forces, which narrows fingers to a singular point, and surface tension, 

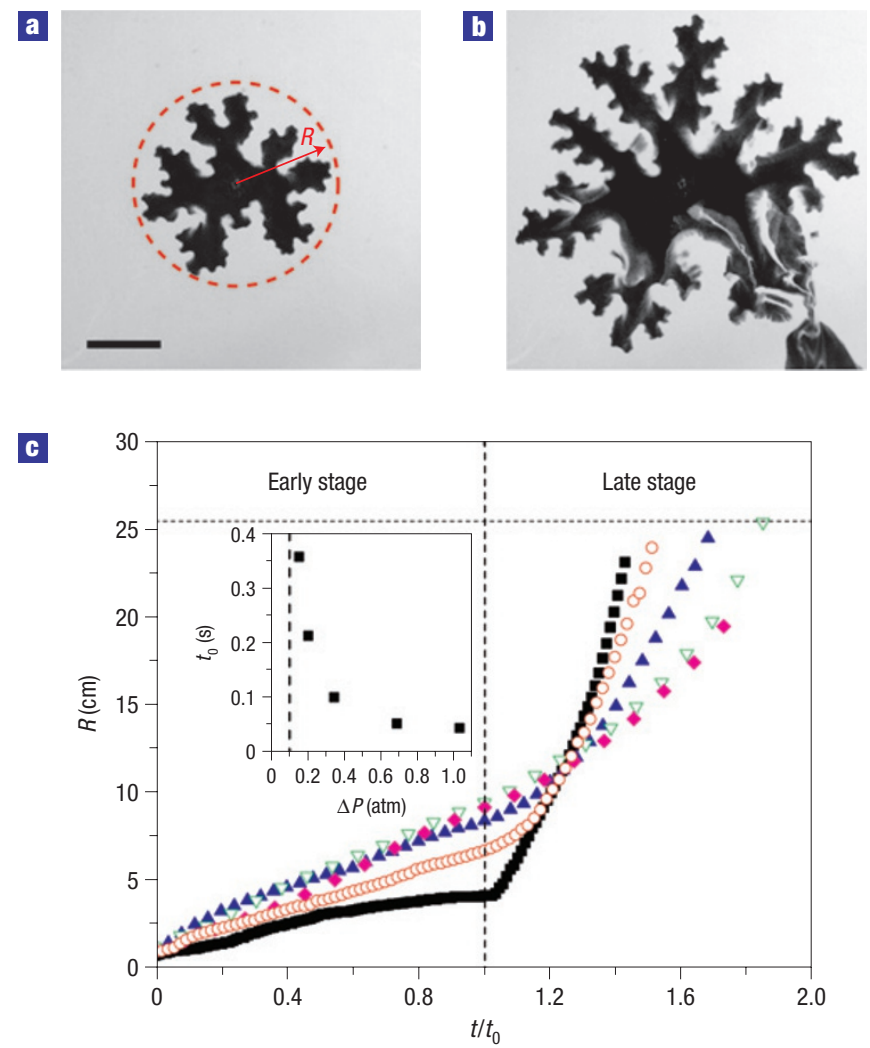

Figure 1 Granular fingering. a,b, Finger patterns during the early stage, $t=0.08 \mathrm{~s}$ (a), and the late stage, $t=0.168 \mathrm{~s}(\mathbf{b})$, of growth. The grey regions in the images are still-undisturbed glass beads and the black regions are empty areas where the gas has displaced the beads. The data were obtained with beads of diameter $d=107 \mu \mathrm{m}$, plate separation $b=0.51 \mathrm{~mm}$, system diameter $L=50.8 \mathrm{~cm}$ and fixed gas overpressure $\Delta P=0.48 \mathrm{~atm}$. The scale bar is $3.0 \mathrm{~cm}$. $R$ is defined as the radius of the longest finger measured from the central hole, as shown by the dashed circle in a. c, $R$ versus renormalized time $t / t_{0}$. The early and late stage are separated at $t=t_{0}$. Symbols from left (black square) to right (pink diamond) in the late stage are $\Delta P=0.15 \mathrm{~atm}, 0.20 \mathrm{~atm}, 0.34 \mathrm{~atm}, 0.68 \mathrm{~atm}$ and $1.02 \mathrm{~atm}$ respectively. The horizontal dotted line marks the boundary of the cell. Inset: The time $t_{0}$ as a function of $\Delta P$. It seems to diverge at $\Delta P_{\text {th }}$ (vertical dashed line) where patterns no longer grow.

which blunts sharp structures in the interface, determines the characteristic length scale of the pattern. Hence, decreasing the driving pressure, which slows down pattern growth, results in blunter patterns in fluid fingering ${ }^{6}$.

As in newtonian fluid fingering, granular fingering grows through tip-splitting instead of side-branching ${ }^{2}$. However, rather than exhibiting an obvious characteristic length scale as found in fluids, the granular fingers widen during growth. To quantify the growth, we define the characteristic width of our pattern, $W$, as the width of fingers just before splitting, as shown in the lower inset of Fig. 2 d. We measure $W$ versus the local interface velocity, $V$, at the tip of fingers for systems with different gap thickness, $b$, and plate diameter, $L$, and with different granular material density $\rho$ and size $d$. The results of these studies are shown in the upper inset of Fig. 2d. As shown, higher velocities produce wider fingers. The main panel of Fig. 2d shows that we can collapse all data onto a single curve with a scaling $W \sim(V L d)^{1 / 2} \rho^{1 / 4}$. Compared with the scaling of classical fluid fingering, equation (1), the width of
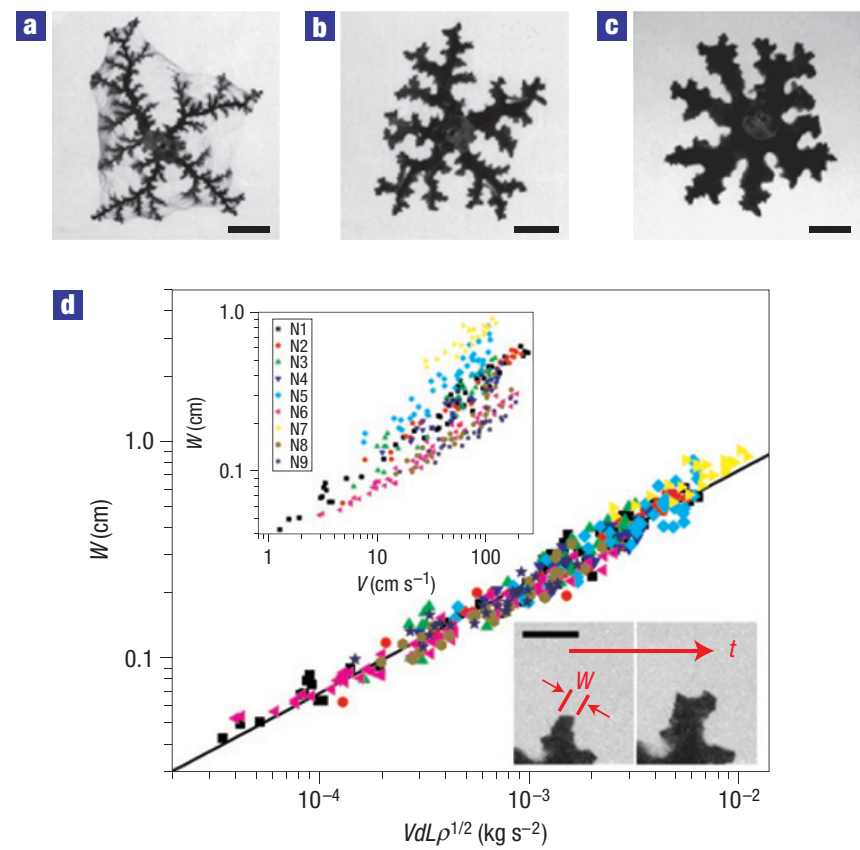

Figure 2 Scaling of the granular fingering instability. a-c, Patterns at different overpressure $\Delta P$ with $d=54 \mu \mathrm{m}, b=0.25 \mathrm{~mm}$ and $L=50.8 \mathrm{~cm}$.

$\Delta P=0.10 \mathrm{~atm}(\mathbf{a}), 0.20 \mathrm{~atm}(\mathbf{b}), 1.36 \mathrm{~atm}(\mathbf{c})$. The scale bars are $1.5 \mathrm{~cm}$. Tips of fingers at low pressure are much finer and sharper than those at high pressure. Similarly, the pattern is more like DLA near $\Delta P_{\text {th }}=0.09$ atm. $\mathbf{d}$, Scaling of finger width, $W$, versus $V L d \rho^{1 / 2}$. The black line is the best power-law fit, with slope $0.51 \pm 0.01$. Lower inset: Growth and tip-splitting of fingers at $\Delta P=0.15 \mathrm{~atm}$ with $d=107 \mu \mathrm{m}, b=0.51 \mathrm{~mm}$ and $L=50.8 \mathrm{~cm}$. The scale bar is $0.8 \mathrm{~cm}$. The time between two images is $0.028 \mathrm{~s}$. As shown, $W$ is defined as the finger width just before it splits. Notice the sharp cusp shown in the second picture during the growth of a finger. Upper inset: Finger $W$ versus tip velocity $V$. Data shown are for $d=107 \mu \mathrm{m}$ glass beads $\left(\rho=2.6 \mathrm{~g} \mathrm{~cm}^{-3}\right)$ with $L=50.8 \mathrm{~cm}$ and $b=0.254 \mathrm{~mm}$ (N1), $L=50.8 \mathrm{~cm}$ and $b=0.508 \mathrm{~mm}$ (N2), $L=50.8 \mathrm{~cm}$ and $b=0.762 \mathrm{~mm}$ (N3), $L=50.8 \mathrm{~cm}$ and $b=1.143 \mathrm{~mm}$ (N4), $L=25.3 \mathrm{~cm}$ and $b=0.508 \mathrm{~mm}$ (N8), $L=17.5 \mathrm{~cm}$ and $b=0.508 \mathrm{~mm}$ (N9); for $d=54 \mu \mathrm{m}$ glass beads with $L=50.8 \mathrm{~cm}$ and $b=0.254 \mathrm{~mm}$ (N6); for $d=359 \mu \mathrm{m}$ glass beads with $L=50.8 \mathrm{~cm}$ and $b=1.143 \mathrm{~mm}$ (N7) and for $d=130 \mu \mathrm{m}$ copper beads $\left(\rho=8.4 \mathrm{~g} \mathrm{~cm}^{-3}\right)$ with $L=50.8 \mathrm{~cm}$ and $b=0.508 \mathrm{~mm}$ (N5).

granular fingers grows as $V^{1 / 2}$ rather than decreasing as $V^{-1 / 2}$ and it is independent of the gap thickness $b$. Furthermore, the parameters of the granular particles themselves, the size and density of the beads, enter the scaling.

To quantify global features of patterns at different $\Delta P$, we calculate their fractal dimension by measuring the number of boxes, $N$, needed to cover the entire pattern, as a function of the size of the box, $l$. The analysed patterns are taken from the end of the early stage. We find that the dimension of patterns, $D$, defined as the power of $N \sim l^{D}$, is limited between that of a circle, $D=2$, and that of a diffusion-limit-aggregation (DLA) pattern ${ }^{29}, D=1.70 \pm 0.02$.

In the zero-surface-tension limit, viscous fingering patterns are predicted to have the same fractal dimension as DLA (refs 2,7,18,19). As we decrease the driving pressure towards the threshold pressure, $\Delta P_{\text {th }}$, we find that $D$ approaches the exponent characteristic of DLA. This suggests that granular fingering may only show the zero-surface-tension-fluid behaviour or the anticipated singularity near the threshold pressure, $\Delta P_{\text {th }}$. To investigate this regime in detail, we conducted a constant flow 

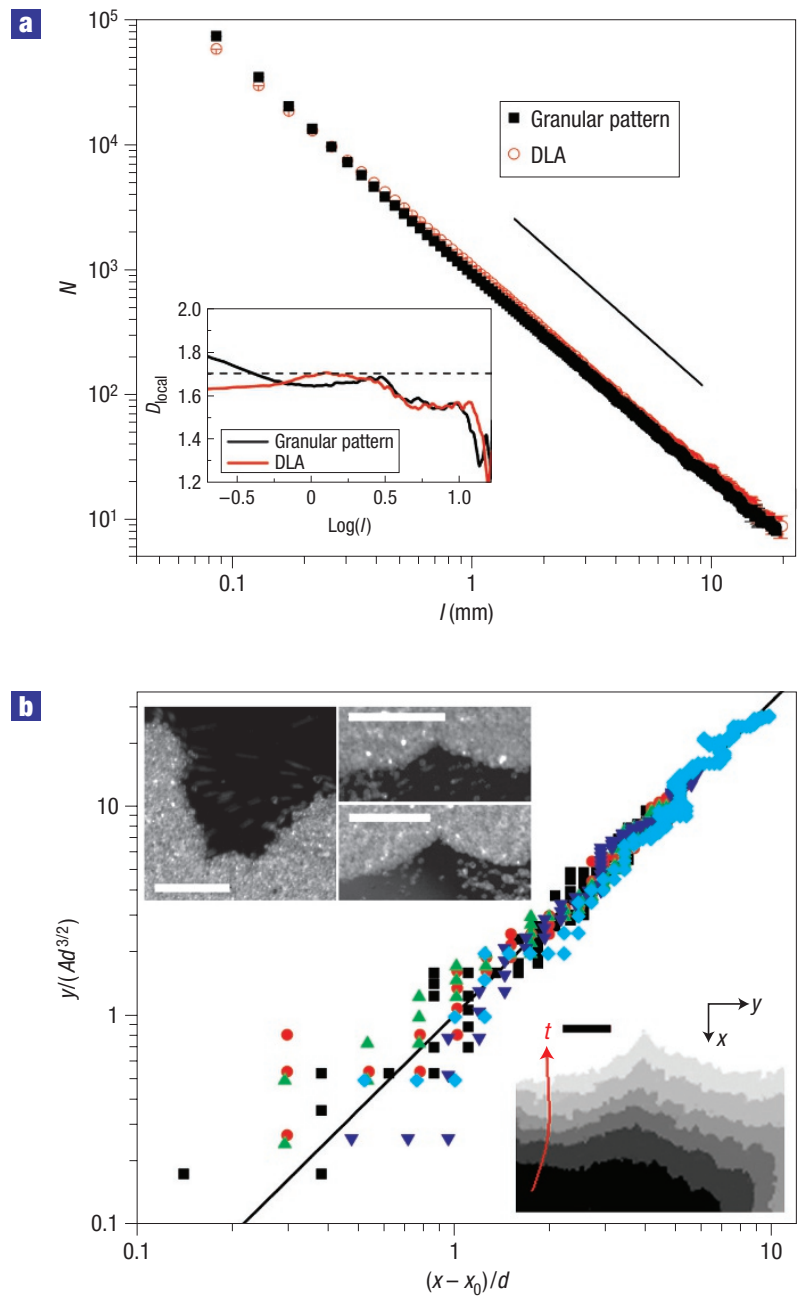

Figure 3 Singular dynamics of granular fingering. a, Comparison between a granular fingering pattern near $\Delta P_{\text {th }}$ and DLA. Shown are box-counting results for a pattern generated under constant flow rate conditions $(d=54 \mu \mathrm{m}$ and $b=0.25 \mathrm{~mm}$ ) and for a DLA pattern generated numerically with the same resolution as that of the granular pattern. $N$ is the number of boxes of side length / needed to cover a pattern. Inset: Local slope of the box-counting results, corresponding to the local fractal dimension, $D_{\text {local }}$, as computed by a linear least-squares fit over the sliding interval $\Delta \log (I)=0.45$. The local slope of our pattern at constant flow rate matches that of the DLA pattern except for the smallest box sizes. The dimension of our DLA pattern is a little smaller than the literature value ${ }^{29} 1.70 \pm 0.02$ owing to the finite resolution. $\mathbf{b}$, Cusp structure in the interface. Upper inset: Three representative images of the air-sand interface for $d=107 \mu \mathrm{m}, b=0.51 \mathrm{~mm}$ and $L=50.8 \mathrm{~cm}$. The scale bars are $1.5 \mathrm{~mm}$. Individual grains are seen in the pictures. Lower inset: Time evolution of an individual cusp. The scale bar is $0.5 \mathrm{~mm}$. Main panel: Scaling of the cusp profiles. Data shown are combined from different experiments, from different cusps in the same experiment, and from left and right arms of each cusp. Vertical and horizontal axes are in units of particle size, $d$, and the $y$ axis is normalized by the free parameter $A$ (see text). Note that we follow the theoretical papers ${ }^{21-23}$ that define the $x$ axis as the growth direction so that the cusp tip coincides with $y=0$. The line shows a power law with slope $3 / 2$. We identified the cusp structure before the growth of almost every finger for small particles (54 $\mu \mathrm{m}$ and $107 \mu \mathrm{m})$ near $\Delta P_{\text {th }}$.

rate experiment as described above. The fractal dimension of the resulting pattern $(D=1.68 \pm 0.02)$ indeed overlaps with that of DLA as seen in Fig. 3a.
We can also focus on a small region along the interface and observe cusp formation as seen in the upper inset of Fig. 3b. These cusp tips can be sharp down to the grain level. Cusp formation occurs just before each finger starts to grow. These cusps are sharp and different from any structures found in newtonian fluids where the finger tips are always round owing to surface tension ${ }^{4-6}$. Theory based on the laplacian growth of conventional fluid fingering in the zero-surface-tension limit suggests ${ }^{2,17,21-23}$ that the shape of the profile at the singularity should scale as $y=A\left(x-x_{0}\right)^{3 / 2}$, where $A$ is a free parameter and $x=x_{0}$ is the position of the singularity. We can collapse the experimental profiles at the time when they are sharpest, onto a single master curve: $y=A\left(x-x_{0}\right)^{\beta}$ with $\beta=1.43 \pm 0.20$ as shown in Fig. 3b. Although the $\beta=3 / 2$ scaling was not derived with dense granular fluids in mind, it is consistent with our granular results at the instant of cusp formation. The lower inset of Fig. 3b shows the time evolution of an individual cusp. However, owing to the stick-slip motion of the interface and to the rapid movement of the finger tips, we cannot resolve the time scaling of the tip velocity.

Because of the absence of surface tension and the irrelevance of thermal energy in granular material, the interfacial patterns formed in our system do not relax when we turn off the driving pressure. Without mechanical perturbation, patterns such as those shown in Fig. 2 retain their sharp structure. Hence, our system provides an opportunity to study the steady-state structure of the patterns. This is in contrast to other systems where surface tension and thermal diffusion always smooth out the interface $\mathrm{e}^{30}$.

Theoretically, it has been known that fluid fingering in the zero-surface-tension limit would lead to singular cusp structure locally and DLA fractal geometry globally ${ }^{2,17-23}$. Nevertheless, the connection between the local singular dynamics and global fractal shape remains far from clear. Despite its unique properties such as compressibility, permeability to gas and interparticle friction, granular flow nevertheless provides the first experimental evidence for the coexistence of these two singular features and shows how the local cusps evolve into a global fractal. Surprisingly, theories based on the hydrodynamics of normal fluids give a good description of the singularity formation observed here in the two-phase dense granular flow. However, we note that the particle diameter, $d$, enters the scaling of granular fingers as an important parameter. Without surface tension to provide a scale to regularize the singularity, the grain diameter may provide that scale.

Received 21 September 2007; accepted 10 December 2007; published 27 January 2008

References

1. Taylor, G. I. \& Saffman, P. G. The penetration of a fluid into a porous medium or Hele-Shaw cell containing a more viscous fluid. Proc. R. Soc. A 245, 312-329 (1958).

2. Bensimon, D., Kadanoff, L. P., Liang, S., Shraiman, B. I. \& Tang, C. Viscous flows in two dimensions. Rev. Mod. Phys. 58, 977-999 (1986).

3. Homsy, G. M. Viscous fingering in porous media. Ann. Rev. Fluid Mech. 19, 271-311 (1987).

4. Paterson, L. Radial fingering in a Hele-Shaw cell. J. Fluid Mech. 113, 513-529 (1981).

5. Praud, O. \& Swinney, H. Fractal dimension and unscreened angles measured for radial viscous fingering. Phys. Rev. E 72, 011406 (2005).

6. Ristroph, L., Thrasher, M., Mineev-Weinstein, M. B. \& Swinney, H. L. Fjords in viscous fingering selection of width and opening angle. Phys. Rev. E 74, 015201(R) (2006).

7. Nittmann, J., Daccord, G. \& Stanley, H. E. Fractal growth of viscous fingers: Quantitative characterization of a fluid instability phenomenon. Nature 314, 141-144 (1985).

8. van Damme, H., Obrecht, F., Levitz, P., Gatineau, L. \& Laroche, C. Fractal viscous fingering in clay slurries. Nature 320, 731-733 (1986).

9. Lindner, A., Bonn, D., Poiré, E. C., Amer, M. B. \& Meunier, J. Viscous fingering in non-Newtonian fluids. J. Fluid Mech. 469, 237-256 (2002).

10. van Damme, H., Lemaire, E., Abdelhaye, Y. O. M., Mourchid, A. \& Levitz, P. in Non-Linearity and Breakdown in Soft Condensed Matter (eds Bardhan, K. K., Chakrabarti, B. K. \& Hansen, A.) (Springer, Berlin, 1993)

11. Johnsen, Ø., Toussaint, R., Måløy, K. J. \& Flekkøy, E. G. Pattern formation during air injection into granular materials confined in a circular Hele-Shaw cell. Phys. Rev. E 74, 011301 (2006).

12. Sandes, B., Knudsen, H. A., Måløy, K. J. \& Flekkøy, E. G. Labyrinth patterns in confined granular-fluid systems. Phys. Rev. Lett. 99, 038001 (2007).

13. Vinningland, J. L., Johnsen, Ø., Flekkøy, E. G., Toussaint, R. \& Måløy, K. J. Granular Rayleigh-Taylor instability: Experiments and simulations. Phys. Rev. Lett. 99, 048001 (2007).

14. Pinto, S. F. et al. Granular fingers on jammed systems: New fluidlike patterns arising in grain-grain invasion experiments. Phys. Rev. Lett. 99, 068001 (2007). 
15. Chevalier, C., Lindner, A. \& Clement, E. Destabilization of a Saffman-Taylor fingerlike pattern in a granular suspension. Phys. Rev. Lett. 99, 174501 (2007).

16. Cheng, X., Varas, G., Citron, D., Jaeger, H. M. \& Nagel, S. R. Collective behavior in a granular jet: Emergence of a liquid with zero surface tension. Phys. Rev. Lett. 99, 188001 (2007).

17. Bertozzi, A. L., Brenner, M. P., Dupont, T. F. \& Kadanoff, L. P. in Trends and Perspectives in Applied Mathematics (ed. Sirovich, L.) (Springer, New York, 1994).

18. Mathiesen, J., Procaccia, I., Swinney, H. L. \& Thrasher, M. The universality class of diffusion limited aggregation and viscous fingering. Europhys. Lett. 76, 257-263 (2006).

19. Patterson, L. Diffusion-limited aggregation and two-fluid displacements in porous media. Phys. Rev. Lett. 52, 1621-1624 (1984).

20. Howison, S. D. Cusp development in Hele-Shaw flow with a free surface. SIAM J. Appl. Math. 46, 20-26 (1986).

21. Teodorescu, R., Wiegmann, P. \& Zabrodin, A. Unstable fingering patterns of Hele-Shaw flows as a dispersionless limit of the Kortweg-de Vries hierarchy. Phys. Rev. Lett. 95, 044502 (2005).

22. Bettelheim, E., Agam, O., Zabrodin, A. \& Wiegmann, P. Singularities of the Hele-Shaw flow and shock waves in dispersive media. Phys. Rev. Lett. 95, 244504 (2005).

23. Bettelheim, E. \& Agam, O. Tip-splitting evolution in the idealized Saffman-Taylor problem. J. Phys. A 39, 1759-1765 (2006).

24. Jaeger, H. M., Nagel, S. R. \& Behringer, R. P. Granular solids, liquids, and gases. Rev. Mod. Phys. 68 $1259-1273(1996)$
25. Conway, S. L., Shinbrot, T. \& Glasser, B. J. A Taylor vortex analogy in granular flow. Nature 431, 433-437 (2004).

26. Yop, P., Forterre, Y. \& Pouliquen, O. A constitutive law for dense granular flows. Nature 441, 727-730 (2006).

27. Aranson, I. S. \& Tsimring, L. S. Patterns and collective behavior in granular media: Theoretical concepts. Rev. Mod. Phys. 78, 641-692 (2006).

28. Albert, R., Albert, I., Hornbaker, D., Schiffer, P. \& Barabási, A.-L. Maximum angle of stability in wet and dry spherical granular media. Phys. Rev. E 56, R6271 (1997).

29. Witten, T. A. \& Sander, L. M. Diffusion-limited aggregation, a kinetic critical phenomenon. Phys. Rev. Lett. 47, 1400-1403 (1981).

30. Sharon, E., Moore, M. G., McCormick, W. D. \& Swinney, H. L. Coarsening of fractal viscous fingering patterns. Phys. Rev. Lett. 91, 205504 (2003).

\section{Acknowledgements}

We thank E. Bettelheim, E. Corwin, S. Y. Lee, J. Royer, P. Wiegmann and L. N. Zou for fruitful discussions. This work was supported by the MRSEC program of the NSF under No. DMR-0213745 and by the W. M. Keck Foundation.

Correspondence and requests for materials should be addressed to X.C.

Reprints and permission information is available online at http://npg.nature.com/reprintsandpermissions/ 